\title{
ÍNDICE $S$ PARA AVALIAÇÃO DA QUALIDADE FÍSICA DE SOLOS ${ }^{(1)}$
}

\author{
Celsemy Eleutério Maia ${ }^{(2)}$
}

\begin{abstract}
RESUMO
O conceito da qualidade física do solo atualmente é bem estabelecido, principalmente pelos índices que avaliam a qualidade do solo. $O$ índice $S$ é um recente indicador da qualidade física do solo relacionado com características importantes do solo. O objetivo deste trabalho foi fazer uma avaliação do método de obtenção do índice $S$ quanto à derivada da função de curva de retenção de água da curva usando ou não a transformação $\ln (h)$, bem como avaliar o problema de unidades e da restrição $m=1-1 / n$ no cálculo do índice. Concluiu-se que o valor do índice $\mathrm{S}$ é superior quando a umidade do solo usada no ajuste da curva de retenção de água proposta por van Genuchten (1980) é expressa na forma volumétrica, quando comparada com a gravimétrica. Para a mesma unidade da umidade do solo, não se observa diferença nos valores do índice $S$ para qualquer unidade de tensão. $O$ valor do índice $S$ difere de acordo com o uso ou não da restrição $m=1-1 /$ n para estimativa dos parâmetros $m$ e $n$ da curva de retenção proposta por van Genuchten (1980).
\end{abstract}

Termos de indexação: curva de retenção de água, qualidade do solo, modelagem.

\section{SUMMARY: USE OF THE S-INDEX TO EVALUATE SOIL PHYSICAL QUALITY}

Nowadays the concept of soil physical quality is well-established, based mainly on the indices that quantify soil quality. The $\mathrm{S}$ index is a new indicator of soil physical quality related to important soil characteristics. The purpose of this study was to evaluate the method of obtaining the $\mathrm{S}$ index and the derivative of the function of the of water retention curve, using the transformation $\ln (\mathrm{h})$ or not, and assess the problem of units and the restriction $m=1-1$ / $n$ for the index calculation. It was concluded that the $\mathrm{S}$ index value is higher when the soil moisture used to adjust the water retention curve of van Genuchten (1980) is expressed as volumetric than as gravimetric moisture. For the same unit of soil moisture, no difference was observed in the $\mathrm{S}$ index for any tension unit. The $\mathrm{S}$ index value differs according to the use or not use of the restriction $m=1-1 / n$ to estimate the parameters $m$ and $\mathrm{n}$ of the van Genuchten retention curve.

Index terms: water retention curve, soil quality, modeling.

(1) Recebido para publicação em 11 de abril de 2011 e aprovado em 13 de setembro de 2011.

(2) Engenheiro-Agrônomo, DS em Recursos Naturais. Professor do Departamento de Ciências Ambientais e Tecnológicas da Universidade Federal Rural do Semiárido - Ufersa. BR 110, km 47, Costa e Silva, CEP 59625-900 Mossoró (RN). Bolsista do CNPq. E-mail: celsemy@ufersa.edu.br 


\section{INTRODUÇÃO}

Para avaliar a qualidade física dos solos, Dexter (2004) propôs um novo método, denominado de índice $S$, baseado no ponto de inflexão do modelo de curva de retenção da água proposta por van Genuchten (1980). Segundo Dexter (2004), maiores valores de $S$ (maior inclinação) indicam melhor distribuição do tamanho de poros, condizente com condições estruturais que estabelecem um adequado funcionamento físico do solo. Assim, fatores relacionados com o solo (textura) e com o manejo (matéria orgânica e estrutura), que influenciam a distribuição do tamanho de poros, refletem em mudanças nos valores de $S$ e, portanto, na qualidade física do solo. Este autor propõe valores de $S>0,035$ e $S<0,035$ para áreas com condições favoráveis e restritivas para o crescimento das raízes das plantas, respectivamente; valores de $S<0,020$ indicam condições físicas do solo muito pobres e com alta restrição ao crescimento das raízes das plantas. Assim, o índice $S$ apresenta sensibilidade para identificar a degradação da qualidade física do solo em diferentes sistemas de uso do solo. O maior valor $S$ na floresta, por exemplo, indicou a melhor configuração de poros no solo e, portanto, menor restrição física para o crescimento de raízes das plantas, seja por aeração, por restrição mecânica ou por características de retenção de água, quando comparado com o de áreas cultivadas (Streck et al., 2008). Entretanto, Silva et al. (2010) afirmam que os valores do índice S proposto por Dexter (2004) são provisórios para classificar a qualidade física dos solos. Afirmam ainda que estudos realizados pela Universidade Estadual de Maringá em 2006 comprovaram a eficiência do índice $\mathrm{S}$ na avaliação da qualidade física do solo sob diferentes sistemas de uso e manejo, porém alertaram que o índice ainda carece de uma quantidade considerável de pesquisas que comprovem a sua eficácia como indicador da qualidade física do solo.

Segundo Dexter (2004), a determinação do valor de $S$ pode ser feita diretamente a partir da curva de retenção de água, com a plotagem da umidade do solo em função do potencial matricial. Entretanto, é mais conveniente ajustar a curva a uma função matemática e, pela derivada, calcular a inclinação no ponto de inflexão com base nos parâmetros da função; isso porque a curva de retenção de água proposta por van Genuchten (1980) apresenta um ponto crítico, observado pela forma gráfica dela quando os valores do módulo do potencial matricial $(h)$ são transformados usando $\ln (h)$. Contudo, igualando a segunda derivada da equação 1 a zero, verifica-se que, mesmo sem a transformação $\ln (h)$, o modelo proposto por van Genuchten (1980) apresenta ponto de inflexão. Nesse sentido, sem a transformação $\ln (h)$, observa-se que a derivada primeira da equação 1 é dada pela equação 2 , diferindo da obtida por Dexter (2004) usando $\ln (h)$ - equação 3.

$$
\theta=\theta r+\frac{\theta s-\theta r}{\left[1+(\alpha h)^{n}\right]^{m}}
$$

em que: $\theta=$ umidade do solo $\left(\mathrm{m}^{3} \mathrm{~m}^{-3}\right.$ ou $\left.\mathrm{kg} \mathrm{kg}^{-1}\right) ; \theta_{s}=$ umidade de saturação $\left(\mathrm{m}^{3} \mathrm{~m}^{-3} \mathrm{ou} \mathrm{kg} \mathrm{kg}^{-1}\right) ; \theta_{r}=$ umidade residual do solo $\left(\mathrm{m}^{3} \mathrm{~m}^{-3}\right.$ ou $\left.\mathrm{kg} \mathrm{kg}^{-1}\right) ; h=$ módulo do potencial matricial ( $\left.\mathrm{MPa}, \mathrm{kPa}, \mathrm{hPa}, \mathrm{cm} \mathrm{H}_{2} \mathrm{O}\right) ; \alpha, n$ e $m=$ parâmetros do modelo, com $a$ em $1 /$ tensão (MPa, $\mathrm{kPa}, \mathrm{hPa}, \mathrm{cm} \mathrm{H}_{2} \mathrm{O}$ ); e $n$ e $m$ são adimensionais.

$$
\frac{d \theta}{d h}=\frac{-m n \alpha^{n}\left(\theta_{s}-\theta_{r}\right) h^{n-1}}{\left[1+(\alpha h)^{n}\right]^{m+1}}
$$

Dexter (2004), para derivar a equação 1 usando $\ln (h)$, fez uma transformação de variável, $\ln (h)=y \rightarrow h=e^{y}$; usando a regra da cadeia $\frac{d \theta}{d y}=\frac{d \theta}{d h} \frac{d h}{d y} \rightarrow \frac{d \theta}{d \ln (h)}=\frac{d \theta}{d h} \frac{d h}{d \ln (h)}$ e sendo $\frac{d \theta}{d h}$ igual à equação $2 \mathrm{e} \frac{d h}{d y}=e^{y}=h$, obtém-se a equação 3 .

$$
\frac{d \theta}{d \ln (h)}=\frac{-m n \alpha^{n}\left(\theta_{s}-\theta_{r}\right) h^{n}}{\left[1+(\alpha h)^{n}\right]^{m+1}}
$$

Os pontos críticos usando valores de $h$ e $\ln (h)$ podem ser observados na figura 1, indicando que nos dois casos existe ponto de inflexão, porém que os valores desse ponto usando $h$ são inferiores quando comparado com $\ln (h)$ para tensão em $\mathrm{kPa}$. O módulo do potencial matricial onde se observa o ponto de inflexão é dado pelas equações 4 e 5 , para dados não transformados ou usando $\ln (h)$, respectivamente, e assumindo a restrição $m=1-1 / n$, para $n>1$.

$$
\begin{aligned}
& h_{p i}=\frac{m^{1 / n}}{\alpha} \\
& h_{p i}=\frac{1}{\alpha}\left(\frac{1}{m}\right)^{1 / n}
\end{aligned}
$$

Conceitualmente, a umidade do solo no potencial matricial igual a zero é a umidade de saturação $\left(\theta_{\mathrm{s}}\right)$, o que não é válido quando utilizada a transformação $\ln (h)$. Além disso, dependendo da unidade da tensão, os valores transformados usando $\ln (h)$ serão negativos quando os valores de tensão estiverem entre 0 e 1 . Nesse caso, seguindo o sistema internacional de unidades, usando a tensão em $\mathrm{MPa}$, os valores variam de 0 até $1,5 \mathrm{MPa}$ e todos os valores entre 0 e 1 apresentam $\ln (h)<0$; quando se usa $\mathrm{kPa}$ (os valores variam de 0 a $1.500 \mathrm{kPa}$ ), a curva desloca-se para o primeiro quadrante dos eixos cartesianos, mas uma parte dela ainda permanece com valores $\operatorname{de} \ln (h)<0$, o que pode ser solucionado quando se usa a tensão em hPa (0 a $15.000 \mathrm{hPa}$ ) (Figura 2), como utilizado por Dexter (2004), porém sem a definição sobre a tensão zero equivaler à umidade de saturação do solo, devido ao problema da descontinuidade da função no ponto $h=0$. 


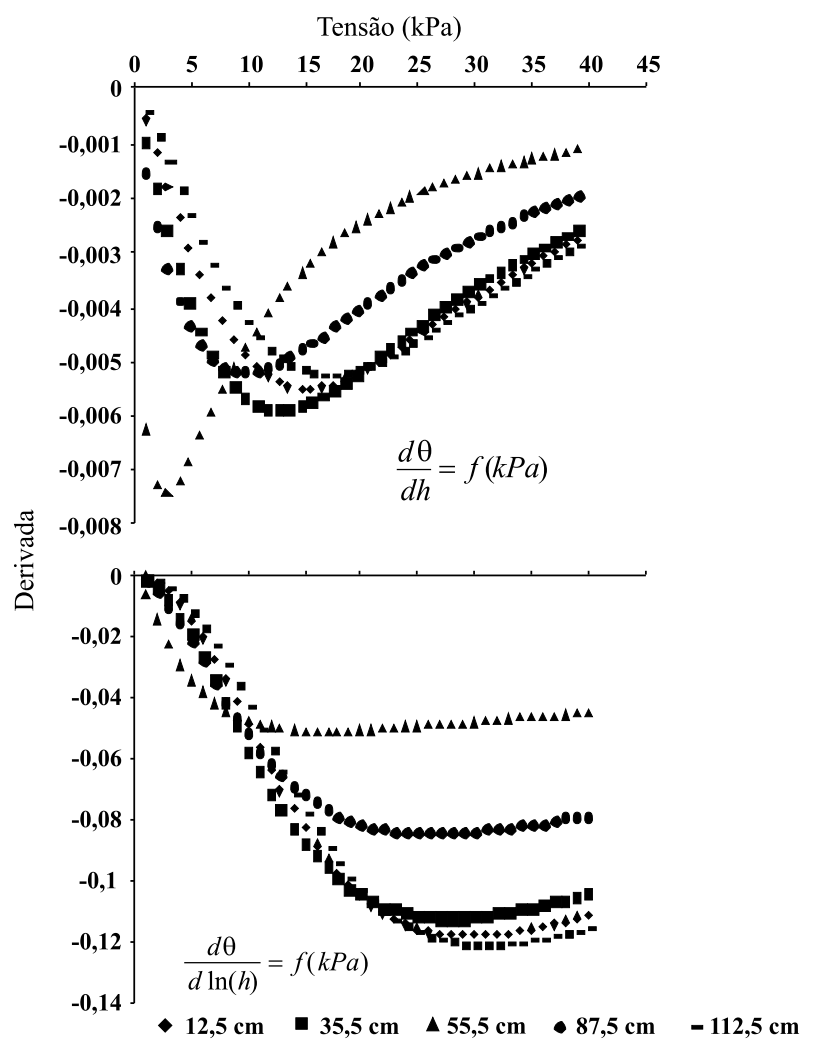

Figura 1. Valores da derivada da equação 1 sem ou com a transformação $\ln (h)$ para diferentes profundidades do solo.

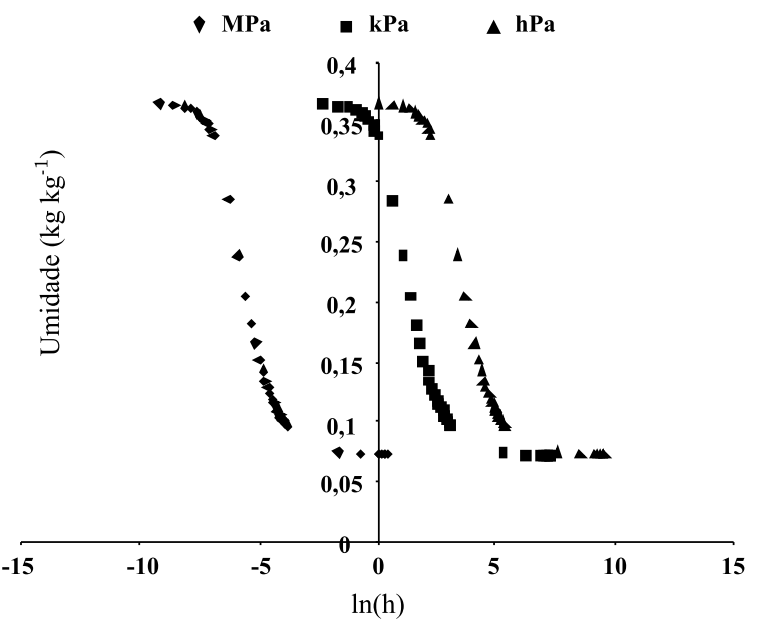

Figura 2. Umidade do solo em função $\ln (h) \operatorname{com} h$ em MPa, kPa e hPa.

Substituindo a equação 4 em 2 e a 5 em 3, têm-se as equações 6 e 7, sendo esta última proposta por Dexter (2004) para o cálculo do índice $S$. Nos dois casos, as equações calculam a declividade da reta tangente ao ponto de inflexão da curva de retenção de água, utilizando os parâmetros do ajuste da curva conforme modelo proposto por van Genuchten (1980).

$$
\begin{gathered}
\frac{d \theta}{d h_{p i}}=-n \alpha(\theta s-\theta r)\left(\frac{m}{m+1}\right)^{m+1} \\
\frac{d \theta}{d h_{p i}}=S=\frac{-n(\theta s-\theta r)}{\left(1+\frac{1}{m}\right)^{m+1}}
\end{gathered}
$$

Observando as equações 6 e 7, levando em consideração que $n$ e $m$ são adimensionais, a unidade da equação 6 é umidade de $\theta$ por unidade de $h$, ao passo que a da 7 é apenas umidade $\left(\mathrm{m}^{3} \mathrm{~m}^{-3}\right.$ ou $\left.\mathrm{kg} \mathrm{kg}^{-1}\right)$ e que, matematicamente, a diferença é o parâmetro $\alpha$ presente no numerador da equação 6 e ausente na 7 . Sendo a unidade de $\alpha$ igual a $h^{-1}$, dividindo a equação 6 por $\alpha$, a unidade passa a ser apenas a da umidade e a equação 6 se iguala a 7 .

Nesse sentido, trabalhando com unidades diferentes, tanto de umidade quanto de tensão, o valor da derivada deve mudar. Mesmo assim, assumindo que a unidade de $S$ é a da umidade do solo, de acordo com a equação 7 , esta deveria ser expressa nos resultados, porém o que se observa é que o índice $S$ é expresso como se fosse adimensional.

O objetivo deste trabalho foi avaliar o método do índice $S$ quanto à derivada da função de retenção de água da curva proposta por van Genuchten (1980) usando ou não a transformação $\ln (h)$, bem como avaliar o problema de unidades e da restrição $\mathrm{m}=1-$ 1/n no cálculo do índice.

\section{MATERIAL E MÉTODOS}

Os dados utilizados neste trabalho foram obtidos de Moreira \& Silva (1987), localizado na Unidade de Execução e Pesquisa (UEP) da Empresa Pernambucana de Pesquisa Agropecuária (IPA), no município de Goiana, PE, com caracterização granulométrica de acordo com o quadro 1.

Com os dados das amostras indeformadas, foram ajustadas as curvas de retenção de água para cada camada do solo usando o modelo proposto por van Genuchten (1980), de acordo com a equação 1 , assumindo ou não a restrição $\mathrm{m}=1-1 / \mathrm{n}$ e usando a umidade do solo em $\mathrm{m}^{3} \mathrm{~m}^{-3}$ ou kg kg-1 e as tensões $(h)$

Quadro 1. Teores de areia, silte e argila e densidade do solo por camada de um Argissolo VermelhoAmarelo

\begin{tabular}{ccrrr}
\hline Camada & Areia & Silte & Argila & Ds \\
\hline m & & $\mathrm{g} \mathrm{kg}^{-1}$ & & $\mathrm{Mg} \mathrm{m}^{-3}$ \\
\cline { 2 - 4 } $0-0,25$ & 895 & 47 & 58 & 1,56 \\
$0,25-0,46$ & 825 & 49 & 126 & 1,59 \\
$0,46-0,65$ & 687 & 44 & 269 & 1,55 \\
$0,65-1,10$ & 690 & 51 & 259 & 1,48 \\
$1,10-1,55$ & 660 & 55 & 285 & 1,48 \\
\hline
\end{tabular}

Fonte: Moreira \& Silva (1987). 
em $\mathrm{MPa}, \mathrm{kPa}, \mathrm{hPa}$ e $\mathrm{cmH}_{2} \mathrm{O}$. O índice $S$ foi calculado pela equação 7 , e a declividade no ponto de inflexão sem a transformação $\ln (h)$, pela equação 6 .

\section{RESULTADOS E DISCUSSÃO}

Ajustando os dados ao modelo de retenção de água usando como variável dependente a umidade $\mathrm{em} \mathrm{m}^{3} \mathrm{~m}^{-3}$ e kg kg-1, e como variável independente a tensão de água no solo ( $h$ ), observa-se (Quadro 2) que os valores do índice $S$ em módulo foram diferentes, sendo superiores quando a umidade foi expressa $\mathrm{em} \mathrm{m}^{3} \mathrm{~m}^{-3}$, e que estes não variaram com a unidade da tensão na mesma profundidade. Isso implica, para comparação dos índices $S$ em diferentes estudos, as umidades devem ser expressas na mesma unidade $\left(\mathrm{m}^{3} \mathrm{~m}^{-3} \mathrm{ou}\right.$ $\mathrm{kg} \mathrm{kg}^{-1}$ ). Dexter \& Bird (2001), avaliando o ponto de inflexão na curva de retenção, trabalharam com as unidades de $\mathrm{hPa}$ e $\mathrm{kg} \mathrm{kg}^{-1}$, para tensão e umidade do solo, respectivamente - mesmas unidades utilizadas por Dexter (2004) para propor o índice $S$. Outros autores também calcularam o índice utilizando essas unidades, como Reynolds et al. (2009). A diferença do índice $S$ calculado usando $\mathrm{m}^{3} \mathrm{~m}^{-3}$ ou $\mathrm{kg} \mathrm{kg}^{-1}$ é matematicamente observada na equação 7 . Considerando que, no ajuste dos dados para uma mesma unidade de tensão, os valores de $\alpha, n$ e $m$ não se alteram, o mesmo não acontece com as umidades $\theta_{s}$ e $\theta_{r}$, que são inferiores quando expressas em $\mathrm{kg} \mathrm{kg}^{-1}$, comparadas com a umidade em $\mathrm{m}^{3} \mathrm{~m}^{-3}$, alterando o

Quadro 2. Valores estimados de $\theta_{s}, \theta_{r}, \alpha, n$ e $m$, coeficiente de determinação $\left(\mathrm{R}^{2}\right), d \theta / d h_{p i}$ e índice $S$, para umidade $\mathrm{em} \mathrm{m}^{3} \mathrm{~m}^{-3} \mathrm{e} \mathrm{kg} \mathrm{kg}^{-1}$ e para diferentes unidades de tensão na curva de retenção, assumindo a restrição $m=1-1 / n$ para cinco camadas de solo em amostras indeformadas

\begin{tabular}{|c|c|c|c|c|c|c|c|c|c|c|}
\hline & \multicolumn{10}{|c|}{ Umidade } \\
\hline & \multicolumn{10}{|c|}{ Camada (m) } \\
\hline & $0-0,25$ & $0,25-0,46$ & $0,46-0,65$ & $0,65-1,10$ & $1,10-1,15$ & $0-0,25$ & $0,25-0,46$ & $0,46-0,65$ & $0,65-1,10$ & $1,10-1,15$ \\
\hline & \multicolumn{5}{|c|}{$m^{3} m^{-3}$} & \multicolumn{5}{|c|}{$-\mathrm{kgkg}^{-1}$} \\
\hline & \multicolumn{10}{|c|}{ Tensão em MPa } \\
\hline$\theta_{s}$ & 0,5700 & 0,6199 & 0,5839 & 0,5911 & 0,5966 & 0,3654 & 0,3899 & 0,3767 & 0,3994 & 0,4031 \\
\hline$\theta_{r}$ & 0,1115 & 0,1308 & 0,1936 & 0,1941 & 0,1761 & 0,0715 & 0,0823 & 0,1249 & 0,1312 & 0,1190 \\
\hline$\alpha$ & 467,17 & 527,23 & 1458,3 & 618,39 & 433,59 & 467,184 & 527,231 & 1458,26 & 618,39 & 433,59 \\
\hline$n$ & 2,0581 & 1,9261 & 1,3986 & 1,7526 & 2,1461 & 2,0581 & 1,9261 & 1,3985 & 1,7526 & 2,1461 \\
\hline$m$ & 0,51411 & 0,48082 & 0,285 & 0,4294 & 0,534 & 0,51411 & 0,48082 & 0,28495 & 0,42942 & 0,534 \\
\hline $\mathrm{R}^{2}$ & 0,9944 & 0,9918 & 0,9879 & 0,9948 & 0,9992 & 0,9943 & 0,9918 & 0,9879 & 0,9948 & 0,9992 \\
\hline$d \theta / d h_{p i}$ & $-85,90$ & $-93,90$ & $-114,9$ & $-77,12$ & $-77,54$ & $-55,07$ & $-59,05$ & $-74,14$ & $-52,10$ & $-52,38$ \\
\hline \multirow[t]{2}{*}{$S$} & $-0,1839$ & $-0,1781$ & $-0,0790$ & $-0,1250$ & $-0,1790$ & $-0,1179$ & $-0,1120$ & $-0,0508$ & $-0,0843$ & $-0,1208$ \\
\hline & \multicolumn{10}{|c|}{ Tensão em kPa } \\
\hline$\theta_{s}$ & 0,5700 & 0,6199 & 0,5839 & 0,5911 & 0,5966 & 0,3654 & 0,3899 & 0,3767 & 0,3994 & 0,4031 \\
\hline$\theta_{r}$ & 0,1115 & 0,1308 & 0,1936 & 0,1941 & 0,1761 & 0,0715 & 0,0823 & 0,1249 & 0,1312 & 0,1190 \\
\hline$\alpha$ & 0,4672 & 0,5272 & 1,4583 & 0,6184 & 0,4336 & 0,4672 & & & 0,6184 & 0,4336 \\
\hline$n$ & 2,0581 & 1,9261 & 1,3986 & 1,7526 & 2,1461 & 2,0581 & 1,9261 & 1,3986 & 1,7526 & 2,1461 \\
\hline$m$ & 0,51411 & 0,48082 & 0,285 & 0,4294 & 0,534 & 0,51411 & 0,48082 & 0,285 & 0,42942 & 0,534 \\
\hline $\mathrm{R}^{2}$ & 0,9944 & 0,9918 & 0,9879 & 0,9948 & 0,9992 & 0,9944 & 0,9918 & 0,9879 & 0,9948 & 0,9992 \\
\hline$d \theta / d h_{p i}$ & $-0,0859$ & $-0,0939$ & $-0,1149$ & $-0,0771$ & $-0,0775$ & $-0,0551$ & $-0,0591$ & $-0,0742$ & $-0,0521$ & $-0,0524$ \\
\hline \multirow[t]{2}{*}{$S$} & $-0,1839$ & $-0,1781$ & $-0,0788$ & $-0,1247$ & $-0,1788$ & $-0,1179$ & $-0,1120$ & $-0,0508$ & $-0,0843$ & $-0,1208$ \\
\hline & \multicolumn{10}{|c|}{ Tensão em hPa } \\
\hline$\theta_{s}$ & 0,5700 & 0,6199 & 0,5839 & 0,5911 & 0,5966 & 0,3654 & 0,3899 & 0,3767 & 0,3994 & 0,4031 \\
\hline$\theta_{r}$ & 0,1115 & 0,1308 & 0,1936 & 0,1941 & 0,1761 & 0,0715 & 0,0823 & 0,1249 & 0,1312 & 0,1190 \\
\hline$\alpha$ & 0,0467 & 0,0527 & 0,1458 & 0,0618 & 0,0434 & 0,0467 & 0,0527 & 0,1458 & 0,0618 & 0,0434 \\
\hline$n$ & 2,0581 & 1,9261 & 1,3986 & 1,7526 & 2,1461 & 2,0581 & 1,9261 & 1,3986 & 1,7526 & 2,1461 \\
\hline$m$ & 0,51411 & 0,48082 & 0,285 & 0,4294 & 0,534 & 0,51411 & 0,48082 & 0,285 & 0,42942 & 0,5340 \\
\hline $\mathrm{R}^{2}$ & 0,9944 & 0,9918 & 0,9879 & 0,9948 & 0,9992 & 0,9944 & 0,9918 & 0,9879 & 0,9948 & 0,9992 \\
\hline$d \theta / d h_{p i}$ & $-0,0086$ & $-0,0094$ & $-0,0115$ & $-0,0077$ & $-0,0078$ & $-0,0055$ & $-0,0059$ & $-0,0074$ & $-0,0052$ & $-0,0052$ \\
\hline \multirow[t]{2}{*}{$S$} & $-0,1839$ & $-0,1781$ & $-0,0788$ & $-0,1247$ & $-0,1788$ & $-0,1179$ & $-0,1120$ & $-0,0508$ & $-0,0843$ & $-0,1208$ \\
\hline & \multicolumn{10}{|c|}{ Tensão em cm $\mathrm{H}_{2} \mathrm{O}$} \\
\hline$\theta_{s}$ & 0,5700 & 0,6199 & 0,5839 & 0,5911 & 0,5966 & 0,3654 & 0,3899 & 0,3767 & 0,3994 & 0,4031 \\
\hline$\theta_{r}$ & 0,1115 & 0,1308 & 0,1936 & 0,1941 & 0,1761 & 0,0715 & 0,0823 & 0,1249 & 0,1312 & 0,1190 \\
\hline$\alpha$ & 0,0452 & 0,05104 & 0,1412 & 0,0599 & 0,042 & & 0,05104 & 0,1412 & 0,0599 & 0,0420 \\
\hline$n$ & 2,0581 & 1,9261 & 1,3986 & 1,7526 & 2,1461 & 2,0581 & 1,9261 & 1,3986 & 1,7526 & 2,1461 \\
\hline$m$ & 0,51411 & 0,48082 & 0,2850 & 0,4294 & 0,5340 & 0,51411 & 0,48082 & 0,2850 & 0,42942 & 0,5340 \\
\hline $\mathrm{R}^{2}$ & 0,9944 & 0,9918 & 0,9879 & 0,9948 & 0,9992 & & & & & 0,9992 \\
\hline$d \theta / d h_{p i}$ & $-0,0083$ & $-0,0091$ & $-0,0111$ & $-0,0075$ & $-0,0075$ & $-0,0053$ & $-0,0057$ & $-0,0072$ & $-0,0050$ & $-0,0051$ \\
\hline$S$ & $-0,1839$ & $-0,1781$ & $-0,0788$ & $-0,1247$ & $-0,1788$ & $-0,1179$ & $-0,1120$ & $-0,0508$ & $-0,0843$ & $-0,1208$ \\
\hline
\end{tabular}


numerador da equação 7 e, consequentemente, o índice $S$ (Quadro 2). Apesar de os valores de $S$ em módulo calculados usando $\mathrm{m}^{3} \mathrm{~m}^{-3}$ terem sido maiores quando comparados com aqueles em $\mathrm{kg} \mathrm{kg}^{-1}$, é importante salientar que isso se deve ao fato de a densidade do solo $(D s)$ ser superior a $1,0 \mathrm{Mg} \mathrm{m}^{-3}$ para todas as camadas avaliadas. Dessa maneira, têm-se valores dos índices $S$ superiores em $\mathrm{m}^{3} \mathrm{~m}^{-3}$, quando comparados com os em kg kg-1 , e isso ocorre se $D s>$ $1,0 \mathrm{Mg} \mathrm{m}^{-3}$, e inferiores se $D s<1,0 \mathrm{Mg} \mathrm{m}^{-3}$.

O uso da unidade seria importante para efeito de comparação dos índices. Como matematicamente o índice $S$ pode ser determinado dividindo $d \theta / d h_{p i}$ por $\alpha$, sendo $d \theta / d h_{p i}$ dado em kg kg-1 $\mathrm{kPa}^{-1}$ e $\alpha$ em $\mathrm{kPa}^{-1}$, isso demonstra também que a unidade do índice $S$ é a unidade de umidade, no caso, $\mathrm{kg} \mathrm{kg}^{-1}$, que ao ser multiplicada pela densidade do solo resultaria na expressão do índice em $\mathrm{m}^{3} \mathrm{~m}^{-3}$.

Comparando os valores do índice $S$ com relação à mudança na unidade de tensão $(h)$, observa-se (Quadro 2) que eles não variaram com as tensões avaliadas ( $\mathrm{MPa}, \mathrm{kPa}, \mathrm{hPa}$ e $\mathrm{cm} \mathrm{H}_{2} \mathrm{O}$ ), porém, matematicamente, era de se esperar uma mudança, como observado para os valores de $d \theta / d h_{p i}$. Independentemente da unidade de tensão, os valores do índice $S$ foram sempre negativos, mas, por conveniência, muitos autores adotam o valor absoluto de S, como recomendado por Dexter (2004).

Ainda com relação à unidade de $h$, avaliando os valores de $d \theta / d h_{p i}$, estes foram maiores em valores absolutos quando utilizado o $\mathrm{MPa}$, diminuindo o valor da inclinação no ponto de inflexão na sequência: $\mathrm{MPa}$
$>\mathrm{kPa}>\mathrm{hPa} \cong \mathrm{cm} \mathrm{H}_{2} \mathrm{O}$, tanto para umidade em $\mathrm{m}^{3} \mathrm{~m}^{-3}$ ou $\mathrm{kg} \mathrm{kg}^{-1}$.

Outra forma de avaliar a transformação $\ln (h)$ foi ajustando os valores de umidade em função de $\ln (h)$ utilizando a curva de retenção proposta por van Genuchten (1980) e estimando os valores de $\theta_{s}, \theta_{r}, \alpha$, $n$ e $m$, para obtenção de $d \theta / d h_{p i}$ e do índice $S$, de acordo com as equações 6 e 7 , respectivamente. Nesse sentido, observa-se no quadro 3 que os valores de $d \theta$ / $d h_{p i}$ assemelham-se aos de $S$ do quadro 2 .

Outro ponto importante a ser observado no cálculo do índice $S$ é a restrição proposta por Mualem (1976), que é indicada no ajuste dos dados ao modelo da curva de retenção proposta por van Genuchten (1980). Assim, no quadro 4, observa-se que para a umidade, tanto em $\mathrm{m}^{3} \mathrm{~m}^{-3}$ quanto em $\mathrm{kg} \mathrm{kg}^{-1}$, o valor do índice $S$ alterou quando comparado com os de $S$ do quadro 1 . Assim, é necessário indicar para o cálculo do índice $\mathrm{S}$ o uso da restrição $m=1-1 / n$, pois ela pode apresentar valores diferentes com ou sem uso dessa restrição no cálculo do índice $S$. Como exemplos, Streck et al. (2008) e Silva et al. (2008) não mencionam a restrição, mas comparam seus resultados com os de outros autores. O valor do índice $S$ de 0,035 , proposto por Dexter (2004) como ponto divisor para separar solos com problemas de degradação, foi calculado usando a umidade do solo em $\mathrm{kg} \mathrm{kg}^{-1}$ e a restrição $\mathrm{m}=1-1 / \mathrm{n}$, para solos com $D_{s}>1,0 \mathrm{Mg} \mathrm{m}^{-3}$. Anteriormente ao trabalho de Dexter (2004), Dexter \& Bird (2001) já haviam trabalhado com a umidade em kg kg-1 e com a restrição $m=1-1 / \mathrm{n}$ para o cálculo do ponto de inflexão na curva de retenção.

Quadro 3. Valores estimados de $\theta_{\mathrm{s}}, \theta_{\mathrm{r}}, \alpha$, n e m, coeficiente de determinação $\left(\mathrm{R}^{2}\right), d \theta / d h_{p i}$ e índice $S$, para umidade em m$~^{3} \mathrm{~m}^{-3}$ e $\mathrm{kg} \mathrm{kg}^{-1}$ e para tensão em hPa e cm $\mathrm{H}_{2} \mathrm{O}$ na curva de retenção, usando $\ln (h)$ e a restrição $m=1-1 / n$, para cinco camadas de solo

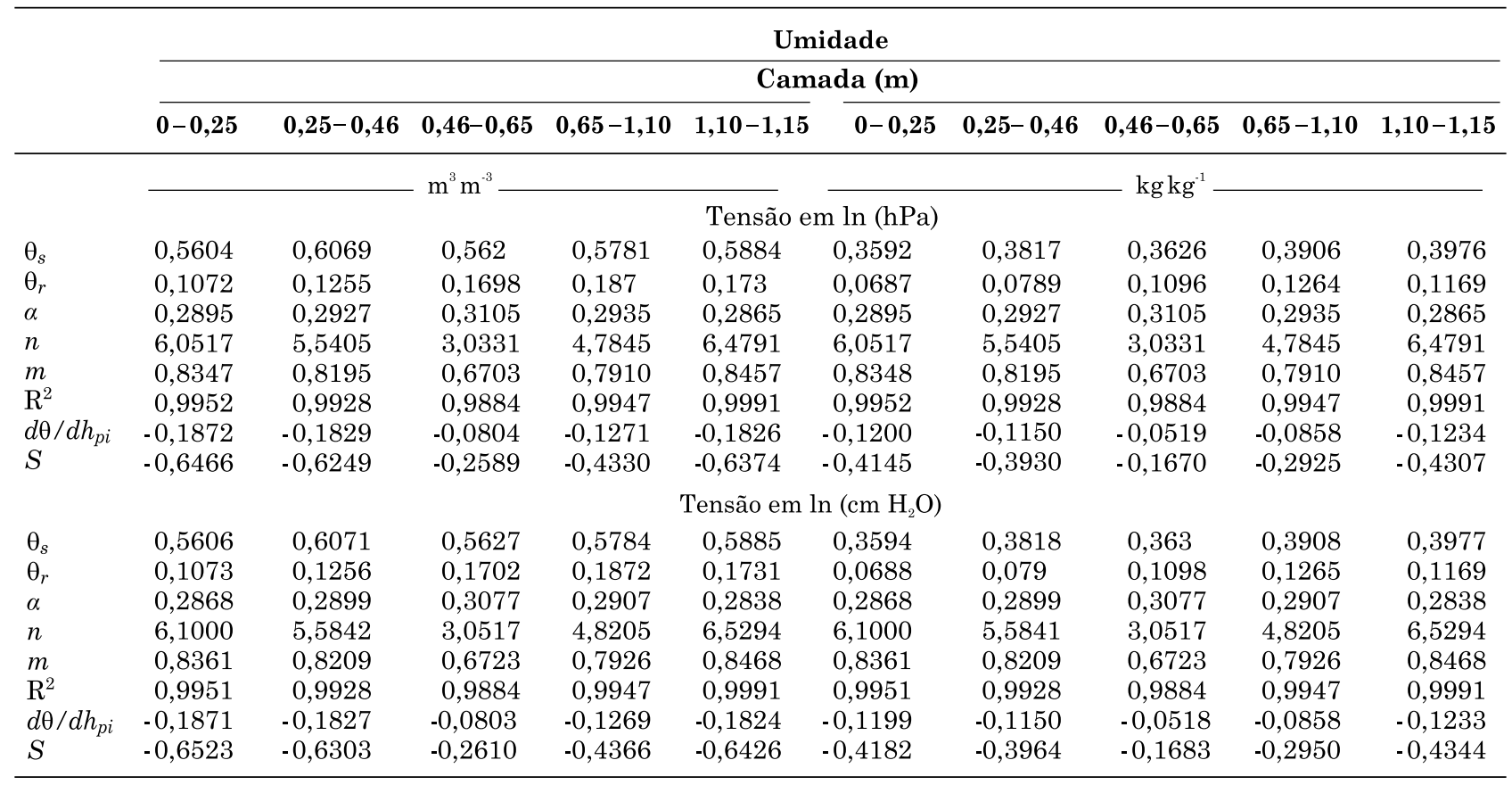


Quadro 4. Valores estimados de $\theta_{\mathrm{s}}, \theta_{\mathrm{r}}, \alpha, \mathrm{n}$ e $\mathrm{m}$, coeficiente de determinação $\left(\mathrm{R}^{2}\right), d \theta / d h h_{p i}$ e índice $S$, para umidade em $\mathrm{m}^{3} \mathrm{~m}^{-3} \mathrm{e} \mathrm{kg} \mathrm{kg} \mathrm{e}^{-1}$ para tensão em MPa e hPa na curva de retenção sem a restrição $\mathrm{m}=1-1 /$ n, para cinco camadas de solo

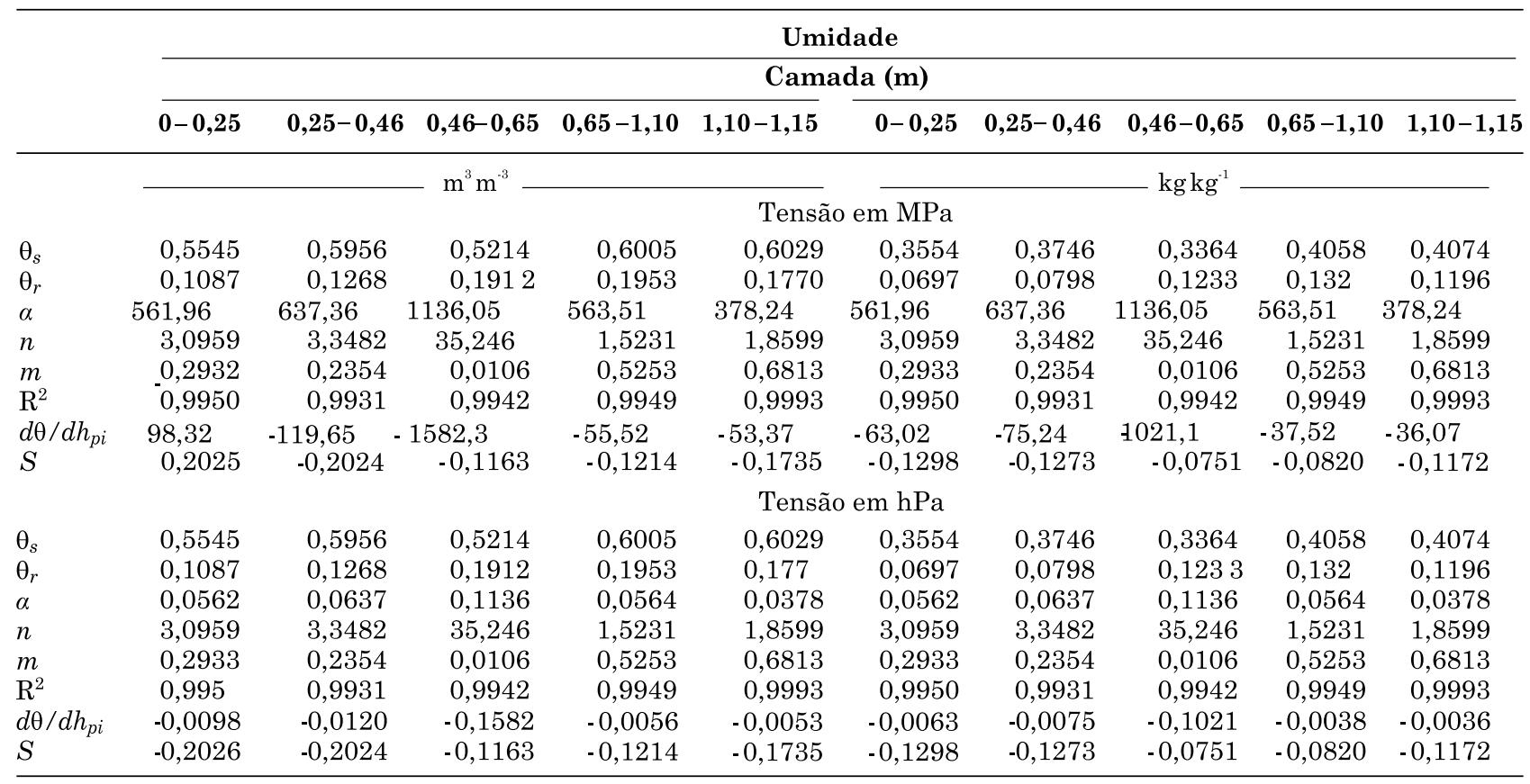

Observando os valores do índice $S$ com base na umidade em $\mathrm{m}^{3} \mathrm{~m}^{-3}$ e $\mathrm{kg} \mathrm{kg}^{-1}$, para as camadas do solo analisadas, verifica-se para a mesma camada discrepância nos valores do índice $S$ de acordo com as unidades dos dados de entrada e da restrição, sendo mais pronunciada a diferença dos valores de $\mathrm{S}$ na camada mais superficial (Quadro 5). Assim,

Quadro 5. Índice $S$ calculado com ou sem a restrição $\mathrm{m}=1-1 / \mathrm{n}$ e umidade do solo $\mathrm{em} \mathrm{m}^{3} \mathrm{~m}^{-3} \mathrm{e} \mathrm{kg} \mathrm{kg}^{-1}$ para diferentes camadas do Argissolo VermelhoAmarelo

\begin{tabular}{|c|c|c|}
\hline \multirow{2}{*}{ Condição } & \multicolumn{2}{|c|}{ Umidade } \\
\hline & $\mathbf{m}^{3} \mathbf{m}^{-3}$ & $\mathrm{~kg} \mathrm{~kg}^{-1}$ \\
\hline \multicolumn{3}{|c|}{ Camada de $0-0,25 \mathrm{~m}$} \\
\hline $\begin{array}{l}\text { Com a restrição } m=1-1 / n \\
\text { Sem a restrição } m=1-1 / n\end{array}$ & $\begin{array}{l}-0,1839 \\
-0,2026\end{array}$ & $\begin{array}{l}-0,1179 \\
-0,1298\end{array}$ \\
\hline \multicolumn{3}{|c|}{ Camada de $0,25-0,46 \mathrm{~m}$} \\
\hline $\begin{array}{l}\text { Com a restrição } m=1-1 / n \\
\text { Sem a restrição } m=1-1 / n\end{array}$ & $\begin{array}{l}-0,1781 \\
-0,2024\end{array}$ & $\begin{array}{l}-0,1120 \\
-0,1273\end{array}$ \\
\hline \multicolumn{3}{|c|}{ Camada de $0,46-0,65 \mathrm{~m}$} \\
\hline $\begin{array}{l}\text { Com a restrição } m=1-1 / n \\
\text { Sem a restrição } m=1-1 / n\end{array}$ & $\begin{array}{l}-0,0788 \\
-0,1163\end{array}$ & $\begin{array}{l}-0,0508 \\
-0,0751\end{array}$ \\
\hline \multicolumn{3}{|c|}{ Camada de $0,65-1,10 \mathrm{~m}$} \\
\hline $\begin{array}{l}\text { Com a restrição } m=1-1 / n \\
\text { Sem a restrição } m=1-1 / n\end{array}$ & $\begin{array}{l}-0,1247 \\
-0,1214\end{array}$ & $\begin{array}{l}-0,0843 \\
-0,0820\end{array}$ \\
\hline \multicolumn{3}{|c|}{ Camada de $1,10-1,15 \mathrm{~m}$} \\
\hline Com a restrição $m=1-1 / n$ & $-0,1788$ & $-0,1208$ \\
\hline Sem a restrição $m=1-1 / n$ & $-0,1735$ & $-0,1172$ \\
\hline
\end{tabular}

recomenda-se que no cálculo do índice $S$ para amostras indeformadas, a umidade do solo deve ser expressa em kg kg-1 e usando a restrição $\mathrm{m}=1-1 / \mathrm{n}$, como sugerido por Dexter (2004) e usado por Reynolds et al. (2009). No quadro 6 são apresentadas as unidades da umidade, da tensão e o uso ou não da restrição $\mathrm{m}=1$ - 1/n no cálculo do índice $S$, chamando a atenção os trabalhos de Andrade \& Stone (2009a,b), que utilizaram para o cálculo do índice $S$ a umidade do solo em kg kg-1 e $\mathrm{m}^{3} \mathrm{~m}^{-3}$, respectivamente (Quadro 6); contudo, como demonstrado aqui, dependendo da densidade do solo, os valores do índice $S$ diferem com a utilização de umidade com unidade diferente.

Quadro 6. Unidades da umidade do solo, tensão e restrição $m=1-1 / n$ utilizadas por nove autores no cálculo do índice $S$

\begin{tabular}{|c|c|c|c|}
\hline Autor(es) & Umidade & Tensão & Restrição \\
\hline Dexter (2004) & $\mathrm{kg} \mathrm{kg}^{-1}$ & $\mathrm{hPa}$ & Sim \\
\hline Streck et al. (2008) & $\mathrm{kg} \mathrm{kg}^{-1}$ & $\mathrm{kPa}$ & Não ${ }^{(1)}$ \\
\hline Andrade \& Stone (2009a) & $\mathrm{kg} \mathrm{kg}^{-1}$ & $\mathrm{kPa}$ & Sim \\
\hline Fidalski \& Tormena (2007) & $\mathrm{kg} \mathrm{kg}^{-1}$ & $\mathrm{hPa}$ & Sim \\
\hline Andrade \& Stone $(2009 b)$ & $\mathrm{m}^{3} \mathrm{~m}^{-3}$ & $\mathrm{cmH}_{2} \mathrm{O}$ & Sim \\
\hline Beutler et al. (2008) & $\mathrm{kg} \mathrm{kg}^{-1}$ & $\mathrm{hPa}$ & Sim \\
\hline Matias et al. (2009) & $\mathrm{kg} \mathrm{kg}^{-1}$ & $\mathrm{cmH}_{2} \mathrm{O}$ & Sim \\
\hline Silva et al. (2008) & $\mathrm{m}^{3} \mathrm{~m}^{-3}$ & $\mathrm{MPa}$ & Não \\
\hline Aguiar (2008) & $\mathrm{m}^{3} \mathrm{~m}^{-3}$ & $\mathrm{MPa}$ & Sim \\
\hline Reynolds et al. (2009) & $\mathrm{kg} \mathrm{kg}^{-1}$ & $\mathrm{hPa}$ & Sim \\
\hline
\end{tabular}




\section{CONCLUSÕES}

1. Usando a tensão sem transformação logarítmica, verificou-se que a curva de retenção proposta por van Genuchten também apresenta ponto de inflexão semelhante ao observado quando usada a transformação $\ln (h)$, porém os valores dos índices $\mathrm{S}$ são diferentes, tanto para o uso ou não da restrição $m$ $=1-1 / \mathrm{n}$.

2. O valor do índice $\mathrm{S}$ é superior quando a umidade do solo usada no ajuste da curva de retenção de água proposta por van Genuchten (1980) é expressa na forma volumétrica, quando comparada com a gravimétrica.

3. Para a mesma unidade da umidade do solo, não há diferença nos valores do índice S para qualquer unidade de tensão.

4. $\mathrm{O}$ uso ou não da restrição $\mathrm{m}=1-1 / \mathrm{n}$ para estimar os parâmetros $m$ e $n$ da curva de retenção de água proposta por van Genuchten (1980) confere diferente valores de índice $\mathrm{S}$.

5. Há necessidade de padronização no uso da umidade em $\mathrm{kg} \mathrm{kg}^{-1}$ e uso da restrição $\mathrm{m}=1-1 / \mathrm{n}$ para comparação dos valores de referência sugeridos por Dexter (2004) para avaliar a qualidade física dos solos, usando o índice S.

\section{LITERATURA CITADA}

AGUIAR, M.I. Qualidade física do solo em sistemas agroflorestais. Viçosa, MG, Universidade Federal de Viçosa, 2008. 79p. (Tese de Mestrado)

ANDRADE, R.S. \& STONE, L.F. Índice $\mathrm{S}$ como indicador da qualidade física de solos do Cerrado brasileiro. R. Bras. Eng. Agri. Amb., 13:382-388, 2009a.

ANDRADE, R.S. \& STONE, L.F. Uso do índice S na determinação da condutividade hidráulica não-saturada de solos do cerrado Brasileiro. R. Bras. Eng. Agrí. Amb. 13:376-381, 2009b.

BEUTLER, A.N.; FREDDI, O.S.; LEONE, C.L. \& CENTURION, J.F. Densidade do solo relativa e parâmetro "S" como indicadores da qualidade física para culturas anuais. $\mathrm{R}$. Bio. Ci. Terra, 8:27-36, 2008.
DEXTER, A.R. Soil physical quality. Part I. Theory, effects of soil texture, density, and organic matter, and effects on root growth. Geoderma, 20:201-214, 2004.

DEXTER, A.R. \& BIRD, N.R.A. Methods for predicting the optimum and the range of soil water contents for tillage based on the water retention curve. Soil Tillage Res., 57:203-212, 2001.

FIDALSKI, J. \& TORMENA, C.A. Homogeneidade da qualidade física do solo nas entrelinhas de um pomar de laranjeira com sistemas de manejo da vegetação permanente. R. Bras. Ci. Solo, 31:637-645, 2007.

MATIAS, S.S.R.; BORBA, J.A.; TICELLI, M.; PANOSSO, A.R. \& CÂMARA, F.T. Atributos físicos de um Latossolo Vermelho submetido a diferentes usos. R. Ci. Agron., 40:331-338, 2009

MOREIRA, J.A.A. \& SILVA, C.J.C.G. Característica de retenção de água de um solo Podzólico Vermelho-Amarelo de Goiana, Pernambuco. Pesq. Agropec. Bras., 22:411418, 1987.

MUALEM, Y. A new model for predicting the hydraulic conductivity of unsaturated porous media. Water Res. Res., 12:513-522, 1976.

REYNOLDS, W.D.; DRURY,C.F.; TAN, C.S.; FOX, C.A. \& YANG, X.M. Use of indicators and pore volume-function characteristics to quantify soil physical quality. Geoderma, 152:252-263, 2009.

SILVA, F.F.; FREDDI, O.S.; CENTURION, J.F.; ARATANI, R.G.; ANDRIOLI, F.F. \& ANDRIOLI, I. Propriedades físicas de um Latossolo Vermelho cultivado no sistema plantio direto. Irriga, 13:191-204, 2008.

SILVA, A.P.; TORMENA, C.A.; DIAS JÚNIOR, M.S.; IMHOFF, S. \& KLEIN, V.A. Indicadores da qualidade física do solo. In: JONG van LIER, Q., ed. Física do solo. Viçosa, MG, Sociedade Brasileira de Ciência do Solo, 2010. p.541-281.

STRECK, C.A.; REINERT, D.J.; REICHERT, J.M. \& HORN, R. Relações do parâmetro $\mathrm{S}$ para algumas propriedades físicas de solos do sul do Brasil. R. Bras. Ci. Solo, 32:26032612, 2008. Número Especial.

van GENUCHTEN, M.T. A closed-form equation for predicting the hydraulic conductivity of unsatured soil. Soil Sci. Soc. Am. J., 44:892-898, 1980. 
